\title{
Estereotipos de género y violencia encubierta de pareja en hombres y mujeres de 18 a 24 años
}

\author{
Gender and violence stereotypes couple covered in men and women from \\ 18 to 24 years old
}

\author{
Alberto Quintana Peña ${ }^{1}$, Alex Grajeda Montalvo ${ }^{2}$, Carmela Malaver Soto ${ }^{3}$, \\ Nicolás Medina Curi ${ }^{4}$, William Montgomery Urday ${ }^{5}$, y Gabriela Ruiz Sánchez ${ }^{6}$ \\ Universidad Nacional Mayor de San Marcos \\ Giancarlo Ojeda Mercado ${ }^{7}$ \\ Universidad Peruana Cayetano Heredia
}

Recibido: $13-12-18$

Aceptado: $17-12-19$

\section{Resumen}

Esta investigación tuvo como objetivos determinar las características de los estereotipos de género en la muestra seleccionada según sexo. Identificar creencias emergentes respecto al género, relaciones de pareja y violencia; y finalmente establecer si la violencia encubierta de pareja, en hombres y mujeres presenta diferencias significantes. Para ello se usó la técnica asociativa de las redes semánticas naturales, adaptada para el presente trabajo por el grupo de estudio con la finalidad de estimar el grado en que cada sujeto comparte el estereotipo del grupo en relación a determinadas conceptualizaciones acerca de la mujer, relaciones de pareja y violencia contra la mujer. Por otra parte se aplicó la Escala de Violencia Encubierta, para medir las pautas del comportamiento de pareja. Los resultados obtenidos muestran que ambos sexos poseen características muy particulares en los estereotipos estudiados, describiéndose la existencia de creencias distintas a las tradicionales en relación al género, las relaciones de pareja y la violencia. Finalmente no se hallaron diferencias en la violencia encubierta según sexo.

Palabras clave: Estereotipos de género; redes semánticas naturales; violencia de pareja; violencia encubierta.

\footnotetext{
1 Docente principal, Universidad Nacional Mayor de San Marcos. E-mail: aquintanap@unmsm.edu.pe 2 Docente asociado, Universidad Nacional Mayor de San Marcos. E-mail: agrajedam@unmsm.edu.pe 3 Docente asociado, Universidad Nacional Mayor de San Marcos. E-mail: cmalavers@unmsm.edu.pe 4 Docente principal, Universidad Nacional Mayor de San Marcos. E-mail: nmedinac@unmsm.edu.pe 5 Docente asociado, Universidad Nacional Mayor de San Marcos. E-mail: jmontgomeryu@unmsm.edu.pe 6 Docente Posgrado, Universidad Nacional Mayor de San Marcos. E-mail: gabyanet@gmail.com.pe 7 Docente, Universidad Peruana Cayetano Heredia. E-mail: giancarlo.ojeda@upch.pe
}

(c) Los autores. Este artículo es publicado por la Revista de Investigación en Psicología de la Facultad de Psicología, Universidad Nacional Mayor de San Marcos. Este es un artículo de acceso abierto, distribuido bajo los términos de la licencia Creative Commons Atribucion - No Comercia_Compartir Igual 4.0 Internacional. (http://creativecommons.org/licenses/by-nc-sa/4.0/) que permite el uso no comercial, distribución y reproducción en cualquier medio, siempre que la obra original sea debidamente citada. 


\begin{abstract}
This research aimed to determine the characteristics of gender stereotypes in the sample selected according to sex. Identify emerging beliefs regarding gender, relationships and violence; and finally to establish whether covert violence in couples, in men and women, presents significant differences. For this, the associative technique of natural semantic networks was used, adapted for the present work by the study group in order to estimate the degree to which each subject shares the stereotype of the group in relation to certain conceptualizations about women, relationships of couple and violence against women. On the other hand, the Covert Violence Scale was applied to measure the patterns of couple behavior. The results obtained show that both sexes have very particular characteristics in the stereotypes studied, describing the existence of beliefs different from the traditional ones in relation to gender, relationships and violence. Finally, no differences were found in covert violence according to sex.
\end{abstract}

Keywords: Gender stereotypes; natural semantic networks; couple violence; covert violence.

En investigaciones previas realizadas por parte de los autores consignados en este artículo, (Quintana, Malaver, Montgomery, Medina, Lúcar, Pineda, Barboza y Domínguez, 2016; Quintana, Malaver y Salazar, 2016), estudiaron el abuso psicológico contra la mujer, las representaciones de violencia de pareja y los estereotipos de género, los estilos atribucionales y estrategias de comunicación, obteniendo importantes conclusiones con respecto a cómo estas variables inciden en la invisibilidad de la violencia doméstica, la vulnerabilidad de la mujer y la ineficacia en las estrategias de afronte, sobre el efecto lesivo de su repercusión estructural y sistémica en nuestra sociedad.

Prosiguiendo con esta línea de investigación y utilizando una metodología novedosa que aportará nuevos datos, se relacionan en esta oportunidad los constructos semánticos con la violencia encubierta de pareja a través de ratios cuantitativos, entendiendo que el problema es de plena actualidad dadas las discusiones presentes hoy en día en nuestro país referentes a la llamada "ideología de género", en respuesta a los ajustes a los contenidos del currículo de educación básica regular propuesto por el Ministerio de Educación del Perú (MINEDU) y, en contraposición, las visiones tradicionales de algunos sectores sociales respecto a cómo debe ser la crianza de los hijos en el aspecto psicosexual.

Lo cierto es que hasta hoy las cifras demuestran que el alto índice de agresión en cuanto a violencia física y sexual (Organización Mundial de la Salud, 2014) parece relacionarse con los estereotipos de género construidos a nivel social. El género (masculino y femenino), en palabras de Cafaro (2014a), suele ser confundido con una "esencia inmutable" de las personas según su sexo, constituyendo un principio organizativo básico de la sociedad y de la consideración diferencial de las personas en ella, que preside el aprendizaje de las actitudes y conductas de los ciudadanos (Cantón, Cantón y Cortés, 2011). Como señala Araya (2004), en este marco la educación escolar actual se caracteriza por un sexismo discriminador que utiliza al rol sexual como criterio de atribución de capacidades, valoraciones 
y significados creados en la vida social, sobre el supuesto de que las mujeres son inferiores y se tienen que resignar al papel de encargadas de las tareas domésticas. En el mismo sentido, la atribución de mitos sexuales masculinos y femeninos referentes a la anatomía y la fisiología, al deseo, a la masturbación, a la moral de la mujer, a la menstruación, a la maternidad, etcétera, son frecuentes en el imaginario sociocultural (Cafaro, 2014b).

A su vez, las actitudes y conductas evocadas por ese aprendizaje influyen sobre la violencia intrafamiliar y la dominación de un género por el otro, frecuentemente en el ámbito de la vida de pareja. En este caso, la violencia de pareja alude a "todo tipo de agresión o maltrato entre personas que comparten una relación sentimental, independientemente del grado de compromiso contraído y de la existencia o no de convivencia" (Labrador, Paz, Alonso y FernándezVelasco, 2012, p. 232). Debe considerarse, además, que la violencia contra la pareja asume muchas veces prácticas casi imperceptibles en la vida cotidiana, tanto de perpetración como de victimización (insultar, controlar, manipular, humillar, obstruir), que pueden denominarse genéricamente con el rótulo de "violencia encubierta", que no involucran agresión física, y, por lo tanto, no suelen ser susceptibles de sancionarse penalmente a pesar de producir efectos lesivos a largo plazo, según lo apunta Lazcorz (2015).

El supuesto básico de esta investigación es que el aprendizaje socialmente condicionado de dichas actitudes y conductas ha dado lugar a redes semánticas implícitas compuestas de palabras y representaciones relacionadas entre sí y que en consecuencia, se puede utilizar la técnica de redes semánticas naturales (Figueroa, González y Solís, 1981), adaptada por nuestro grupo de estudio, para explorar el grado de dicha organización del conocimiento en cada individuo, identificar empíricamente el sistema de creencias compartidos por los individuos. En otras palabras, evidenciar que los estereotipos de género se relacionan con el comportamiento emitido frente al género femenino (Vera, Pimentel y Batista de Albuquerque, 2005). Ello permitirá esclarecer los mecanismos ideológicos que, a través de redes semánticas, establecen un marco regulativo para las prácticas de relación en la pareja; a fin de poseer un adecuado diagnóstico de la problemática para posteriormente planificar y promover estrategias educativas que permitan construir comportamientos más asertivos en las personas, tanto agresores como víctimas, y así promover un adecuado comportamiento ciudadano.

De lo dicho se deduce que la justificación principal de este estudio es que la forma más eficaz de enfrentar el problema señalado es mediante su prevención, para lo cual contribuirá la información proporcionada por el presente estudio. Las acciones preventivas son indispensables para combatir los estereotipos negativos de género, construyendo relaciones de pareja empáticas, dialogantes y cooperativas. Sin embargo, para poder planificar acciones preventivas es importante conocer de manera clara y distinta ¿Cuáles son las características de los estereotipos de género 
en la muestra seleccionada según sexo? ¿Qué creencias emergen respecto al género, relaciones de pareja y violencia en la muestra investigada?. ¿Cómo se manifiesta la violencia encubierta de pareja, en hombres y mujeres de la población de estudio?

\section{Objetivos}

Determinar las características de los estereotipos de género en la muestra seleccionada según sexo

Identificar creencias emergentes respecto al género, relaciones de pareja y violencia en la población investigada

Establecer si el comportamiento de violencia encubierta de pareja, en hombres y mujeres de 18 a 24 años presenta diferencias significativas.

\section{Hipótesis}

Los estereotipos de género muestran una correlación directa y significativa entre sí, permitiendo reconstruir el sistema de creencias respecto al género, relaciones de pareja y violencia en la población investigada.

Existen creencias emergentes respecto al género, relaciones de pareja y violencia en la población investigada que difieren de las clásicamente descritas.

La violencia encubierta de pareja no muestra diferencias estadísticamente significativa entre hombres y mujeres de 18 a 24 años.

\section{METODO}

Diseño de investigación

Se trabaja con el tipo de investigación descriptiva correlacional teniendo en cuenta los criterios, según los cuales esta clase de indagación permite explicar los nexos entre dos o más categorías, definiciones o variables en una situación establecida (Hernández, Fernández y Baptista, 2010).

Nivel de investigación

Básica o pura, pues nuestro objetivo es la contribución de componentes teóricos para el desarrollo de conocimientos en la ciencia psicológica. (Sánchez y Reyes (2015).

Variables

V1: Estereotipos de género.

V2: Violencia encubierta de pareja. 
Población

Ciudadanos hombres y mujeres de 18 a 24 años residentes en San Martin de Porres. De acuerdo a la Municipalidad Distrital de San Martin de Porres (2017) la población adulta entre 20 a 60 años constituye 414,779 pobladores, distribuidos en 198,561 hombres y 216,218 mujeres.

Muestra

Muestreo intencional de 200 sujetos representativos de la población.

\section{Técnicas e Instrumentos}

La técnica de redes semánticas naturales (Figueroa, González, y Solís, 1981), permite recolectar datos a través de un auto-reporte grupal. Las palabras o frases incompletas estímulo fueron presentadas a cada sujeto a manera de un registro de asociación libre de palabras o de frases incompletas (Anexo 1) que permite elicitar cinco palabras definidoras de la representación social del género y de violencia de género en la muestra evaluada, indicándoles que estas palabras pueden ser verbos, adverbios, adjetivos, sustantivos, nombres o pronombres, pero no artículos ni proposiciones. A continuación, se les pidió que jerarquicen esas cinco palabras de acuerdo con la cercanía o importancia que tiene cada una de las palabras con la palabra o frase incompleta estímulo: que le asignarán el número uno a la palabra más cercana al estímulo, el dos a la siguiente, y así sucesivamente, hasta agotar todas las palabras en mención (llamadas "definidoras"). Los materiales para llevar a cabo esta tarea consistieron en hojas de color blanco, rosa y azul.

El valor $\boldsymbol{J}$, que representa el total de palabras denominadas como "definidoras" proporcionadas por los participantes, fue de 114 palabras asociadas. Los pesos semánticos oscilaron entre el $100 \%$ y el $23.35 \%$ para el análisis general, $22.61 \%$ en la hoja blanca, $25.56 \%$ en la hoja rosa y $28.05 \%$ en la azul. Este porcentaje permite ver la amplitud de la significatividad que tuvieron los conceptos o palabras para los respondientes.

A partir de estos datos se obtuvieron los indicadores: Valor J: el total de palabras definidoras que fueron generadas por los sujetos. Valor M: la multiplicación que se hace de la frecuencia de aparición por la jerarquía obtenida para cada palabra definidora. Y el Conjunto SAM: las palabras definidoras que hubieran obtenido los mayores valores "M" totales. Con estos últimos datos se obtuvieron las gráficas presentadas en los resultados. Además de ello se obtuvieron los puntajes de desviación de este conjunto que representan las respuestas de cada sujeto, permitiendo asignarle a cada uno de ellos un puntaje que simboliza el grado en que cada sujeto muestra el estereotipo de género correspondiente a la palabra o frase estímulo a fin de correlacionar los estereotipos entre sí. Al ser una técnica cualitativa no se requiere determinar sus propiedades psicométricas, sin 
embargo Grajeda (2019) refiere que esta técnica posee validez empírica en virtud a que distintas investigaciones muestran su utilidad, entre ellas las de Figueroa, González, y Solis, (1981), Zermeña, Arellano, Ramírez (2005), etc. Asimismo refiere que la confiabilidad obtenida por el índice de coincidencia de 2 evaluadores ha sido igual a 0.99 lo que indicaría muy alta consistencia.

La Escala de Violencia Encubierta, elaborada por Lazcorz (2015), establece su validez a través del análisis factorial exploratorio encontrando multidimensionalidad con valores apropiados en sus cinco categorías, que son "coerción, control, inseguridad emocional, infravaloración y evasión de responsabilidades" (ib., p. 320). Asimismo, la validez convergente con la sub escala agresión psicológica del CTS2 ha sido de 0.66, con un NS de 0.01. Este último dato indica que la prueba es consistente, y por lo tanto confiable, a partir de la correlación de pruebas paralelas.

\section{RESULTADOS}

Luego de aplicarse los instrumentos a una muestra representativa poblacional, se procedió a ingresar los datos en tablas para el análisis cuantitativo a través del Paquete estadístico para Ciencias Sociales (SPSS) versión 23.0. Se utilizaron como ratios las medidas de tendencia central, la correlación y la regresión lineal.

Los resultados de la Tabla 1 se pueden interpretar en el sentido de que en la muestra total todas las dimensiones de violencia encubierta guardan una correlación positiva significativa de moderada a fuerte entre ellas y con el puntaje total. Es decir, el instrumento mide consistentemente el constructo de violencia encubierta.

Por otro lado, los resultados reflejados en la tabla 2, obtenidos valorando el grado en que cada uno de los participantes en el estudio comparte el estereotipo del grupo, se observa que los estereotipos no necesariamente guardan una covariación entre sí, sino que esta relación es mínima o inexistente salvo en relación con algunos de ellos; asociaciones que posibilitan un examen complementario enriquecedor de la información brindada por la técnica de Redes Semánticas Naturales, al potenciar las evaluaciones que permitirían habitualmente emplear esta técnica, y que se ensayan al dar cuenta sobre ello en este análisis y discusión de los resultados. En esta tabla, las correlaciones encontradas en los participantes de la muestra total respecto al grado que comparten los estereotipos de género entre sí, es del 30\%. Específicamente, se encuentran en la muestra total correlaciones significativas, aunque débiles, que indican que mientras más consolidado se encuentre el estereotipo de celos (como persona desconfiada, insegura, temerosa), más consolidados se hallan los estereotipos de romance (como una relación de amor, cariño, confianza, pasión y felicidad) y hombre (fuerte, inteligente, trabajador, cariñoso). Se observa además una covariación entre la consolidación 
del estereotipo de romance (como una relación de amor, cariño, confianza, pasión y felicidad) con los estereotipos de sexo (como una relación de amor, placer, intimidad, pasión, deseo), violencia(como una persona que golpea, agrede, mala y que odia) y enamoramiento (como una relación de amor, comprensión, confianza y compromiso). Los resultados también muestran que conforme se consolida el estereotipo de sexo (como una relación de amor, placer, intimidad, pasión, deseo), se consolida el estereotipo de enamoramiento (como una relación de amor, comprensión, confianza y compromiso). Por último, se visualiza que al consolidarse el estereotipo de sexo (como una relación de amor, placer, intimidad, pasión, deseo), asimismo se consolida el estereotipo de mujer (como una persona inteligente, trabajadora, fuerte, amorosa, bella y responsable).

Tabla 1

Escala de violencia encubierta y sus dimensiones

\begin{tabular}{|c|c|c|c|c|c|c|}
\hline & & INFRAVALORACION & COERCION & EVASION & CONTROL & INSEGURIDAD \\
\hline \multirow[t]{3}{*}{$\begin{array}{l}\text { VIOLENCIA } \\
\text { ENCUBIERTA }\end{array}$} & $\begin{array}{l}\text { Coeficiente de } \\
\text { correlación }\end{array}$ &, $595^{* *}$ &, $563^{* * *}$ &, $629^{* * *}$ &, $659^{* *}$ & ,695* \\
\hline & Sig. (bilateral) &, 000 &, 000 &, 000 &, 000 &, 000 \\
\hline & $\mathrm{N}$ & 111 & 111 & 111 & 111 & 111 \\
\hline \multirow[t]{3}{*}{ INFRAVALORACION } & $\begin{array}{l}\text { Coeficiente de } \\
\text { correlación }\end{array}$ & 1,000 &, $368^{* *}$ &, $343^{\text {t*t }}$ &, $\mathbf{4 1 8}^{* *}$ &, $514^{* *}$ \\
\hline & Sig. (bilateral) & 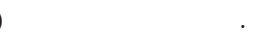 &, 000 &, 000 &, 000 &, 000 \\
\hline & $\mathrm{N}$ & 111 & 111 & 111 & 111 & 111 \\
\hline \multirow[t]{4}{*}{ COERCION } & $\begin{array}{l}\text { Coeficiente de } \\
\text { correlación }\end{array}$ & & 1,000 & ,476 &, $359^{* * *}$ &, $\mathbf{3 8 8}^{* *}$ \\
\hline & Sig. (bilateral) & & . & ,000 &, 000 &, 000 \\
\hline & $\mathrm{N}$ & & 111 & 111 & 111 & 111 \\
\hline & $\begin{array}{l}\text { Coeficiente de } \\
\text { correlación }\end{array}$ & & & 1,000 &, $\mathbf{4 1 3}^{\text {** }}$ &, $\mathbf{4 3 8}^{\text {t*t }}$ \\
\hline \multirow[t]{3}{*}{ EVASION } & Sig. (bilateral) & & & 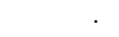 &, 000 &, 000 \\
\hline & $\mathrm{N}$ & & & 111 & 111 & 111 \\
\hline & $\begin{array}{l}\text { Coeficiente de } \\
\text { correlación }\end{array}$ & & & & 1,000 &, $\mathbf{4 8 8}^{\text {t** }}$ \\
\hline \multirow[t]{2}{*}{ CONTROL } & Sig. (bilateral) & & & & . &, 000 \\
\hline & $\mathrm{N}$ & & & & 111 & 111 \\
\hline
\end{tabular}

**. La correlación es significativa en el nivel 0,01 (2 colas).

Tabla 2

Correlación entre los estereotipos de género 


\begin{tabular}{|c|c|c|c|c|c|c|c|}
\hline \multicolumn{2}{|l|}{ Estereotipos } & \multirow{2}{*}{$\begin{array}{c}\text { Hombre } \\
, 092\end{array}$} & \multirow{2}{*}{$\begin{array}{c}\text { Enamorados } \\
-, 025\end{array}$} & \multirow{2}{*}{$\begin{array}{r}\text { Violencia } \\
, \mathbf{1 3 8}^{*}\end{array}$} & \multirow{2}{*}{$\begin{array}{r}\text { Sexo } \\
, 027\end{array}$} & \multirow{2}{*}{$\begin{array}{r}\text { Romance } \\
-, 029\end{array}$} & \multirow{2}{*}{$\begin{array}{r}\text { Celos } \\
, 072\end{array}$} \\
\hline Mujer & $\mathrm{r}$ & & & & & & \\
\hline & Sig. & ,176 & ,715 & ,046 & 691 & 674 & ,302 \\
\hline & $\mathrm{N}$ & 111 & 111 & 111 & 111 & 111 & 111 \\
\hline \multirow[t]{3}{*}{ Hombre } & $\mathrm{r}$ & 1,000 & ,068 & 100 & 042 & 073 &, $197^{\text {k*t }}$ \\
\hline & Sig & . & ,308 & 142 & ,528 & 281 & 005 \\
\hline & $\mathrm{N}$ & 111 & 111 & 111 & 111 & 111 & 111 \\
\hline \multirow[t]{3}{*}{ Enamorados } & $\mathrm{r}$ & & 1,000 & ,104 &, $\mathbf{1 3 3}^{*}$ &, $152^{*}$ & ,100 \\
\hline & Sig & & . & ,129 & ,047 & ,025 & 149 \\
\hline & $\mathrm{N}$ & & 111 & 111 & 111 & 111 & 111 \\
\hline \multirow[t]{3}{*}{ Violencia } & $\mathrm{r}$ & & & 1,000 & ,019 &, $167^{*}$ & ,085 \\
\hline & Sig & & & & ,776 & ,016 & ,227 \\
\hline & $\mathrm{N}$ & & & 111 & 111 & 111 & 111 \\
\hline \multirow[t]{3}{*}{ Sexo } & $\mathrm{r}$ & & & & 1,000 & ,211 & ,055 \\
\hline & Sig & & & & . & ,002 & ,431 \\
\hline & $\mathrm{N}$ & & & & 111 & 111 & 111 \\
\hline \multirow[t]{3}{*}{ Romance } & $\mathrm{r}$ & & & & & 1,000 &, $162^{*}$ \\
\hline & Sig & & & & & . & 021 \\
\hline & $\mathrm{N}$ & & & & & 111 & 111 \\
\hline
\end{tabular}

Se observa que en la medida que se va consolidando el estereotipo de hombre, también se formaliza el de "violencia" y su incidencia en la conformación del estereotipo de "celos", estereotipos que no se condicen con los tradicionales, donde la violencia aparejada con los celos se justificaba como una expresión de amor y manifestación de hombría (Moyeda, Xóchitl, Sánchez y Robles, 2013). Por el contrario, se visualiza al hombre con atributos como"inteligente", "respetuoso" y "responsable", reprobando la violencia con adjetivos de agresión física y vinculándola con emociones de connotación negativa como ira, odio aunado al dolor, lo que se condice con la valoración de los celos asociándolos con desconfianza, inseguridad y también manifestación de agresión como las peleas, encontrándose así cambios en las concepciones clásicas sobre masculinidad (Téllez y Verdú, 2011) (figura 1).

Esto se debería a que el estudio ha considerado una muestra poblacional proveniente del ciclo de vida de la "adultez emergente", constructo acuñado por Arnett (2000) en referencia a estas edades, el cual constituye un fenómeno cultural con aspectos distintivos en función del escenario sociodemográfico de procedencia. Dichos aspectos, relacionados entre otras cosas con variables "tales como el aumento del acceso a la educación universitaria, el aumento de la fuerza laboral, y la postergación de hitos como el matrimonio y tener hijos" (Barrera y 
Vinet, 2017; p. 48), parecen ligarse al empoderamiento de una imagen femenina distinta a la tradicional.

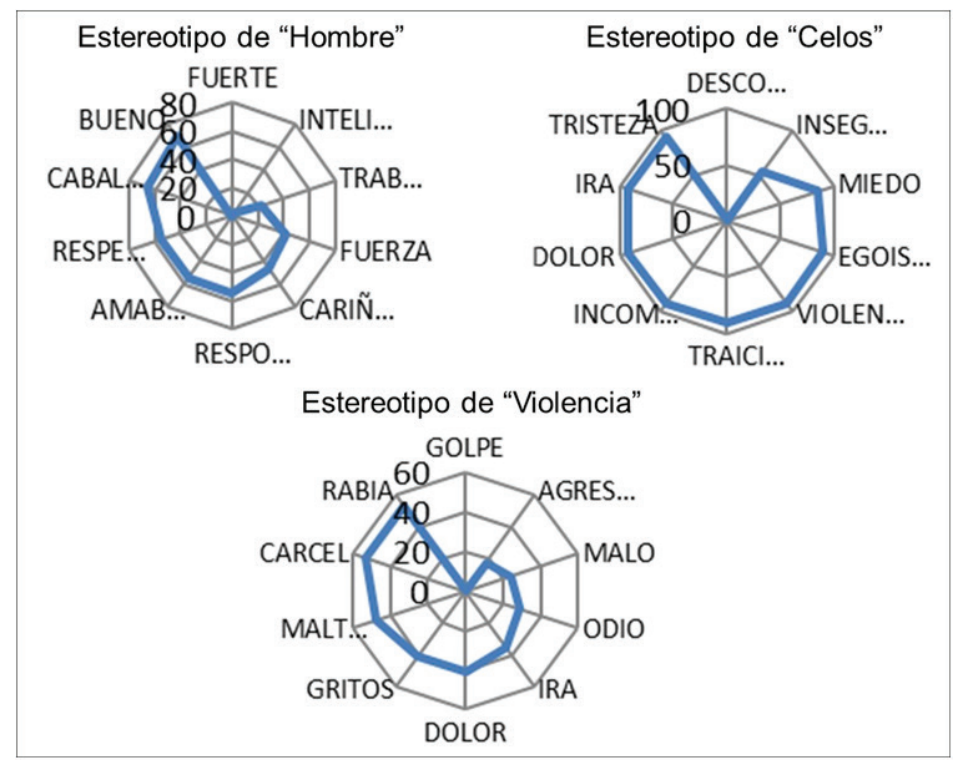

Figura 1. Estereotipos de "Hombre", "Celos" y "Violencia"

Los estereotipos de "mujer bella" y "hombre guapo" (figura 2) se articulan de manera positiva y directa; incluso comparten atributos como "inteligencia", "amabilidad", y se destaca "ternura" y "comprensión" en la mujer; así como también "honestidad" y el atributo físico de "altura" para los hombres, alejándose de los cánones tradicionales de orden estético.

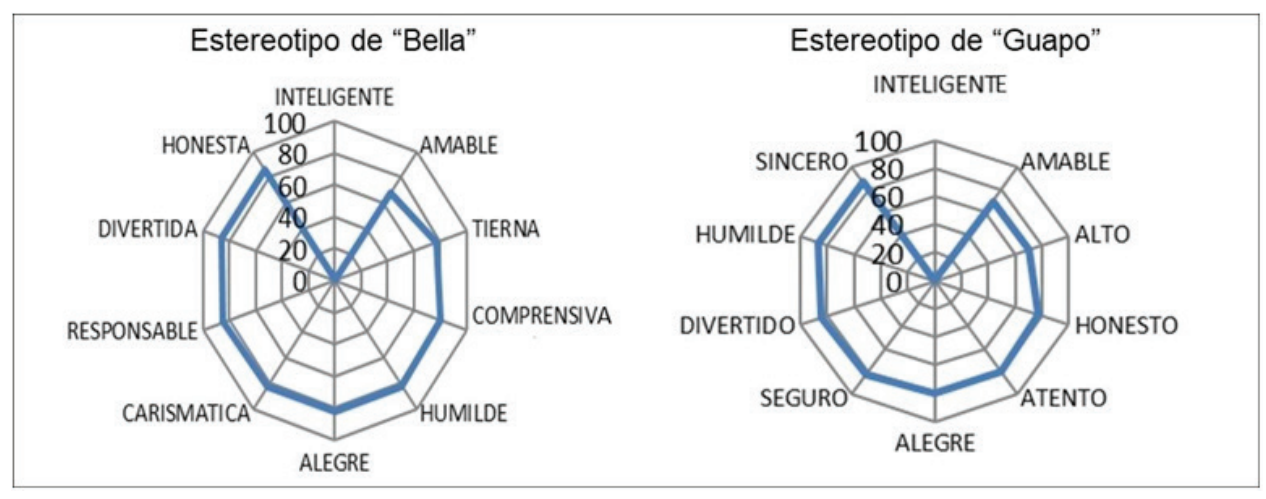

Figura 2. Estereotipos de "Mujer Bella" y "Hombre Guapo" 
Por otro lado, la disminución del "sexo casual": el tener baja o inactividad sexual es más común entre los adultos jóvenes (Tobón, 2012; Twenge, Sherman \& Wells, 2016). Al respecto, el estereotipo de sexo está orientado hacia el amor el placer, la intimidad, el deseo, confianza; por lo cual se liga con mayor fuerza con el "romance" (figura 3) donde el "amor", el "cariño", la "confianza", la "pasión" y la "felicidad" son ejes centrales y abonan hacía la construcción de una relación de pareja, que cuando se desarrolla traspasa las fronteras de la incertidumbre, individualidad, transitoriedad, y apunta hacia la estabilidad (Blandón y López, 2016).

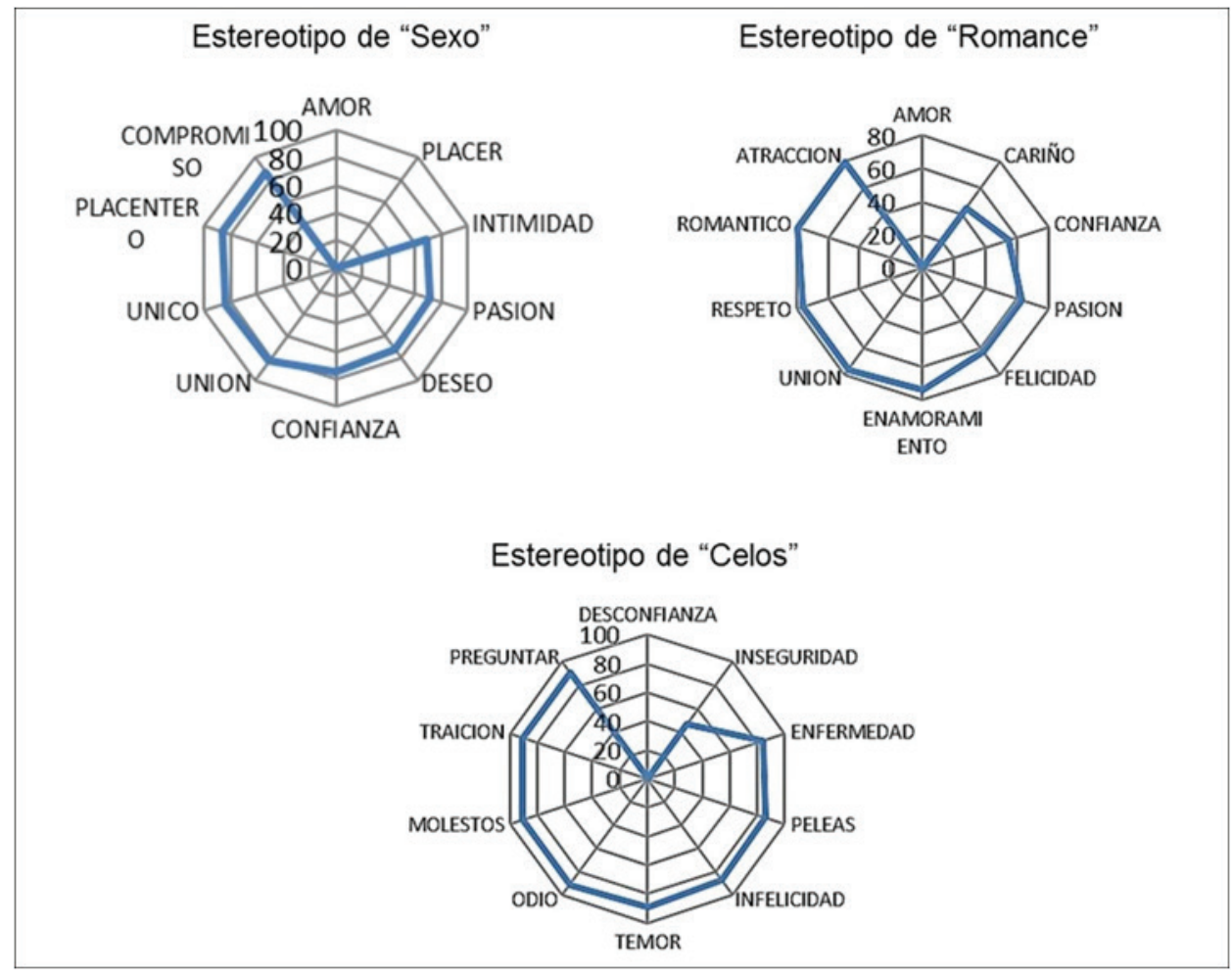

Figura 3. Estereotipos de "Sexo", "Romance" y "Celos"

Se evidencia una relación inversa del estereotipo de enamorados con el grupo etario de estudio, lo cual se ve ratificado por investigaciones anteriores donde se visualiza una dualidad, por un lado puede proyectarse la búsqueda de una vinculación amorosa con compromiso, confianza, comprensión (Blandón y López, 2016), y por otro, la presencia de recelo en el establecimiento de relaciones afectivas, no constituyéndose ellas en una prioridad; para luego optarse por la exploración de relaciones informales (Barrera y Viñet 2017). Con respecto al cambio de percepción de belleza y el estereotipo que se genera, aquel se va 
afianzando con la edad en este grupo etario de los millennials, orientándose más hacia aspectos relacionales como amabilidad, comprensión, ternura, y de competencia como la inteligencia.

Se observa cómo se articulan y comparten atributos los estereotipos de bella y guapo como son inteligencia y sinceridad, además subyacen cualidades para bella como amable y humilde, y en guapo valores como responsabilidad y laboriosidad (trabajador), considerando los aspectos estéticos complementarios para realzar la imagen.

De manera similar sucede correlacionalmente con los estereotipos de hombre y mujer que se centran operativamente en la inteligencia, laboriosidad y fortaleza y relacionalmente en el amor y el cariño. Se ve con esta generación de los millenialls el empoderamiento de la mujer, autopercibiéndose con atributos positivos (Proyecto Scopio, 2015), orientados hacia la equidad de género.

En el sentido de estos modelos de masculinidad y feminidad emergentes es que parece ser necesario consolidar para hacer frente al modelo hegemónico con sus asimetrías de poder y control que permiten su subsistencia (Ruiz 2014).

Tabla 3

Comparación de la violencia encubierta entre los grupos de varones y mujeres

\begin{tabular}{llcc}
\hline \multicolumn{1}{c}{ Hipótesis nula } & \multicolumn{1}{c}{ Prueba } & Sig. & \multicolumn{1}{c}{ Decisión } \\
\hline $\begin{array}{l}\text { La distribución de la violencia } \\
\text { encubierta es la misma entre las } \\
\text { categorías de sexo }\end{array}$ & $\begin{array}{l}\text { Prueba U de Mann- Witney para muestras } \\
\text { independientes }\end{array}$ & 0.951 & Conserve la hipótesis nula \\
\hline
\end{tabular}

La tabla tres nos muestra que no existen diferencias significativas en función al sexo.

\section{DISCUSIÓN}

Como se planteó inicialmente, teniendo a la vista los datos ofrecidos tanto por la técnica de Redes Semánticas Naturales de Figueroa, González, y Solís (1981), como por la Escala de Violencia Encubierta (Lazcorz, 2015), se puede concluir, que los atributos de los estereotipos tradicionales no se revelan en la muestra estudiada, lo que lleva a cuestionar dicha visión sobre los otrora modelos prevalentes de masculinidad y femineidad (Echeburúa y Fernández-Montalvo, 1998; p. 74) y nos permite constatar la emergencia de nuevos modelos, considerando que las feminidades y las masculinidades no se constituyen de una vez y para siempre como entidades fijas, sino que, como señalan Hernández y González (2016), fluyen y cambian con el tiempo a manera de un proceso dinámico. Al respecto, la vulnerabilidad de la masculinidad basada en la represión de sentimientos y emociones para evitar la pérdida de poder, generaría el efecto de que los hombres 
integren a su identidad varonil distintos tipos de significados, normas y roles no tradicionales.

Por otro lado, también está la posibilidad de la "invisibilización" de la violencia por parte de las jóvenes al no considerar comportamientos de maltrato al dominio y al control (García, Fernández, Rodríguez, López, Mosteiro y Lana, 2013) aunado a los mitos sobre el amor romántico que subyacen en la formación de los jóvenes, donde se valida la dependencia, pasividad, cuidado en las mujeres $\mathrm{y}$ en los hombres la conquista, seducción, protección y dominio; justificando manifestaciones de violencia de pareja ante la creencia que el "amor todo lo puede" considerando incluso que algunos comportamientos violentos son una prueba de amor, e incluso los celos y el afán de posesión (Ferrer y Bosch, 2013).

La distribución de las características de los estereotipos de género en la muestra seleccionada respecto a los varones, da como resultado que para ellos el hombre guapo se destaca por ser inteligente amable y alto; que los hombres se ven a sí mismos como inteligentes, respetuosos responsables y fuertes; y que la violencia se caracteriza por golpes, agresión, maldad, odio e ira. Que coexisten con estereotipos emergentes no tan convencionales como considerar entre los atributos de la mujer bella el ser sobre todo inteligente, del sexo estar relacionado al amor y de los celos estar relacionados a la desconfianza e inseguridad enfermiza.

En el caso de las mujeres se ven los mismos estereotipos de características convencionales, como el considerar que los varones se caracterizan por ser fuertes, inteligentes, trabajadores y cariñosos; relacionar los enamorados al amor, respeto y confianza; el romance al amor, cariño y respeto; y relacionar la violencia con los golpes, insultos, maltratos y agresiones. Estas representaciones coexisten con estereotipos emergentes ya no tan convencionales, como considerarse a sí mismas (entre otros atributos) inteligentes, fuertes y valientes, $\mathrm{y}$, al igual que los varones, asociar los celos sobre todo a la desconfianza, la inseguridad y el temor.

Se puede advertir que en la totalidad de la muestra hay correlaciones, aunque débilmente significativas que indican que, a mayor estereotipo de hombres y romance, mayor estereotipo de celos; a mayor estereotipo de enamorados, sexo y violencia, mayor estereotipo de romance; a mayor estereotipo de mujer, mayor estereotipo de violencia; $y$ a mayor estereotipo de enamorados, mayor estereotipo de sexo. En todos estos datos se puede observar la influencia del marco social-virtual que configura esquemas "uniformados" de socialización del género y relaciones interpersonales con base en ciertas cosmovisiones juveniles bastante superficiales, típicas del siglo XXI. A este respecto, es bueno señalar que la influencia de los medios de comunicación tiene un impacto aun no especificado suficientemente sobre la reconfiguración de hábitos en los miembros de la sociedad, y pudiera 
ser que uno de ellos conlleve cierta tendencia a dejar los modelos tradicionales de masculinidad y femineidad y orientarse a una relación de equidad de género como un hábito (véase Vega, 2014).De hecho, como señala Lazcorz (2015), “esas diferencias no se aprecian de la forma tan generalizada como se ha supuesto con frecuencia puesto que los hombres tienden a identificarse con algunos rasgos femeninos en la misma medida que con los masculinos, mientras que las mujeres se afianzan con claridad en los rasgos femeninos, marcando cierta distancia respecto a los masculinos"(p. 313).

En los varones aparecen específicamente correlaciones significativas, aunque débiles, que indican que a mayor estereotipo de hombres y romance mayor estereotipo de celos, y a mayor estereotipo de romance mayor estereotipo de sexo. Esta visión no se halla muy lejos de la mentalidad tradicional vinculada al machismo como posición de dominio (Pazos, Oliva y Hernando, 2014).

Por otra parte, en la muestra femenina se encuentran correlaciones significativas, aunque débiles, que indican que a mayor estereotipo de enamorados y violencia mayor estereotipo de romance; y a mayor estereotipo de mujer y celos mayor estereotipo de hombre. ¿Sería esta una manifestación menor de la telúrica tendencia al "más me pegas, más te quiero"? Si fuera así, aquí se configuraría como una expectativa vagamente generalizada de trato de género. Quizá debiera confrontarse esto con lo que dice Lazcorz (2015) respecto a la violencia encubierta en la muestra española. "Desde el rol de víctima, las mujeres son... más propensas a considerarse receptoras de la inseguridad o los sentimientos de culpa provocados por su pareja" (p. 286).

Finalmente se observa que la violencia encubierta no marca diferencias en función al sexo, contrariamente a lo que se planteaba hipotéticamente que esta sería más fuerte en las mujeres. Al respecto nuestros datos guardan similitud con los presentados en este nuevo milenio por Weisz, Tolman, Callahan, Saunders, \& Black, 2007; y Fernández-González, O’Leary, \& Muñoz-Rivas, 2013; en el sentido que la violencia se distribuye de la misma manera en hombres y mujeres.

\section{CONCLUSIONES}

Se han determinado las características de los estereotipos de género en la muestra seleccionada según sexo encontrándose que estos tienen particularidades muy específicas.

Las creencias emergentes respecto al género, relaciones de pareja y violencia en la población investigada son diferentes a las tradicionales.

Hombres y mujeres de 18 a 24 años presentan puntuaciones promedio similares en violencia encubierta. 


\section{REFERENCIAS}

Arnett, J. J. (2000). Emerging adulthood: A theory of development from the late teens through the twenties.American Psychologist, 55, 469-480.

Araya Umaña, Sandra (2004). Hacia una educación no sexista . Revista Electrónica Actualidades Investigativas en Educación, 4(2), Disponible en: https://www.redalyc. org/articulo.oa?id=447/44740217

Barrera-Herrera, A. y Vinet, E. (2017). Adultez Emergente y características culturales de la etapa en universitarios chilenos. Terapia psicológica, 35(1), 47-56.

Blandón, A.I. y López, L.M. (2016). Comprensiones sobre pareja en la actualidad: jóvenes en busca de estabilidad. Revista Latinoamericana de Ciencias Sociales, Niñez y Juventud, 14 (1), 505-517.

Cafaro, M. E. (2014a). La construcción del género. En A.M. Vega (Comp.). Sexualidades. (pp. 154-161). Buenos Aires: Lugar Editorial.

Cafaro, M. E. (2014b). Mitos sexuales. En A.M. Vega (Comp.). Sexualidades. (pp. 29-40). Buenos Aires: Lugar Editorial.

Cantón, D., Cantón, J. y Cortés, Ma. del R. (2011). Desarrollo de la personalidad y del rol de género. En J. Cantón y Ma. del R. Cortés (Eds.). Desarrollo afectivo y de la personalidad (pp. 153-186). Madrid: Alianza.

Echeburúa, E. y Fernández-Montalvo, J. (1998). Hombres maltratadores. En E. Echeburúa, y P. de Corral (Eds.). Manual de violencia familiar (pp. 72-129). Madrid: Siglo XXI.

Fernández-González, L., O’Leary, K., \& Muñoz-Rivas, M. (2013). We are not joking: Need for controls in reports of dating violence. Journal of Interpersonal Violence, 28, 602-620.

Ferrer, V. y Bosch, E. (2013). Del amor romántico a la violencia de género. Para una coeducación emocional en la agenda educativa. Profesorado. Revista de curriculum y formación de profesorado. 17(1); 105-122.

Figueroa, J. G. González, E. G. y Solis, V.M. (1981). Uma aproximacao ao problema dos significados: As redes semánticas. Revista Latinoamericana de Psicología, 13, 447 458.

García, V.; Fernández, A.; Rodríguez, F.J.; López, M.L.; Mosteiro, M.D. y Lana, A. (2013). Violencia de género en estudiantes de enfermería durante sus relaciones de noviazgo. Atención Primaria, 45(6), 290-296.

Grajeda, A. (2019). Violencia encubierta y estereotipos de género en parejas de adultos jóvenes. Ponencia presentada en el I Congreso Nacional de Estudiantes de Psicología CAEP 2019. Cusco - 2019.

Hernández, R.; Fernández, C y Baptista, P. (2010). Metodología de la investigación. México: Mac Graw Hill s.a.

Hernández, A y Gonzáles, J (2016). Los roles y estereotipos de género en los comportamientos sexuales de jóvenes de Coahuila, México: Aproximación desde la teoría fundamentada. Ciencia Ergo Sum, 23(2). 112-120. 
Labrador, F. J., Paz, P., Alonso, E.y Fernández Velasco,R. (2012). Violencia de pareja. En L.M. Llavona y F.X. Méndez (Eds.), Manual del psicólogo de familia (pp.231-258). Madrid: Pirámide.

Lazcorz, A. (2015). Violencia encubierta en las relaciones de parejas jóvenes. Tesis Doctoral, Departamento de Psicología de la Universidad de Castilla-La Mancha. Recuperado de: https://ruidera.uclm.es/xmlui/bitstream/handle/10578/8675/ TESIS\%20Lascorz\%20Fumanal.pdf?sequence=1

Moyeda, G ; Xóchitl, I; Sánchez, A; Robles y Ojeda, F. (2013) Relaciones entre estilos de amor y violencia en adolescentes Psicología desde el Caribe, 30(2), 211-235.

Municipalidad Distrital de San Martin de Porres (2017). Presupuesto inicial de apertura 2018. Recuperado de http://www.mdsmp.gob.pe/data_files/pia_2018.pdf

Organización Mundial de la Salud (2014). Resumen: Respuesta a la violencia de pareja y a la violencia sexual contra las mujeres. Directrices de la OMS para la práctica clínica y las políticas. Washington, DC: OMS.

Pazos, M.; Oliva, A. y Hernando, Á. (2014). Violencia en relaciones de pareja de jóvenes y adolescentes. Revista Latinoamericana de Psicología, 46(3), 148-159.

Proyecto Scopio, (2015) Índices de desarrollo juvenil. http://www.proyectoscopio.es/

Quintana, A., Malaver, C. y Salazar, M. (2016). Representaciones de violencia de pareja, estereotipo de género, sexismo ambivalente y programas de TV de señal abierta vistos por los adolescentes. Propuesta de investigación al Consejo Superior de Investigaciones de la UNMSM.

Quintana, A., Malaver, C., Montgomery, W., Medina, N., Ruiz, G., Lúcar, F., Pineda, D., Barboza, M. Domínguez,S. (2016). Estilos atributivos y estrategias de comunicación en mujeres con y sin experiencia de abuso psicológico. PsiqueMag, 4(1), 127-149.

Ruiz Repullo, Carmen (2014) Los modelos de atracción en la adolescencia: ¿el triunfo de las identidades hegemónicas? Universidad Pablo de Olavide

Sánchez, H. y Reyes, C. (2015). Metodología y diseños en la investigación científica. Lima: Universidad Ricardo Palma.

Téllez, A., y Verdú, A. (2011). El significado de la masculinidad para el análisis social. Nuevas Tecnologías en Antropología (2), 80-103.

Tobón, J., Vega, M. y Cuervo, J. (2012). Características de la construcción de vínculo afectivo de pareja en la juventud en la ciudad de Medellín. CES Psicología, 5(1), 49-64.

Twenge, J; Sherman, R ; Wells, B (2016). Sexual Inactivity during young adulthood is more common among U.S. millennials and igen: Age, period, and cohort effects on having no sexual partners after age 18. Arch Sex Behav., 46(2):433-440.

Vega, A. M. (2014). ¿Cómo influyen los medios de comunicación en nuestra sexualidad? En A. M. Vega (Comp.). Sexualidades (pp. 359-374). Buenos Aires: Lugar Editorial. 
Vera, J.A., Pimentel, C.A. y Batista de Albuquerque, F.B. (2005). Redes semánticas: Aspectos teóricos, técnicos, metodológicos y analíticos. Ra Ximhai Revista de Sociedad, Cultura y Desarrollo Sustentable, 1(3), 439-451.

Weisz, A., Tolman, R.M., Callahan, M.R., Saunders, D.G., \& Black, B.M. (2007). Informal helpers' responses when adolescents tell them about dating violence or romantic relationship problems. Journal of Adolescence, 30, 853-868. doi: 10.1016/j.adolescence.2006.09.004

Zermeña, A., Arellano, A. y Ramirez, V. (2005). Redes semánticas naturales: técnica para representar los significados que los jóvenes tienen sobre televisión, internet y expectativas de vida. Estudios sobre las Culturas Contemporaneas, diciembre, año/ vol. XI, número 022 Universidad de Colima Colima, México pp. 305-334. 